\section{P.2.20 EPIDEMIC OF BLADDER CANCER IN JAPANESE MALE WORKERS EXPOSED MAINLY TO ORTHO-TOLUIDINE}

${ }^{1}$ Makiko Nakano*, ${ }^{1}$ Kazuyuki Omae, ${ }^{1}$ Yoko Eitaki, ${ }^{1,2}$ Satoko Iwasawa, ${ }^{1}$ Kota Fukai, ${ }^{1,2}$ Noriyuki Yoshioka, ${ }^{3}$ Shigeru Tanaka, ${ }^{4}$ Shigeki Koda, ${ }^{1}$ Toru Takebayashi. ${ }^{1}$ Keio University School of Medicine, Tokyo, Japan; ${ }^{2}$ National Defense Medical College, Saitama, Japan; ${ }^{3}$ Jumonji University, Saitama, Japan; ${ }^{4}$ National Institute of Occupational Safety and Health, Kawasaki, Japan

\subsection{6/OEM-2019-EPI.253}

Background In 2012, ortho-toluidine (OT) was listed as a Group 1 carcinogen by the International Agency for Research on Cancer, based on epidemiologic observations in workers co-exposed to OT and aromatic amines. Between 2014 and 2017, 10 cases of bladder cancer (BC) were identified in Japanese males working at two plants of the same company manufacturing intermediates of organic dyes and pigments.

Objective To describe the features of the $\mathrm{BC}$ epidemic at the plants.

Methods We conducted a cross-sectional study of 76 OT and/ or aromatic amine-exposed workers including the $10 \mathrm{BC}$ cases at the plants in 2017. The co-exposed aromatic amines were para-toluidine, ortho-anisidine, aniline, 2,4-dimethylaniline, and/or ortho-chloroaniline. Details of each worker's job-site histories were obtained from the company records. Past medical symptoms and histories were checked in physician interviews. The subjects were divided into the $\mathrm{BC}$ group $(\mathrm{n}=10)$ and the non-BC group $(n=66)$ and compared. No quantitative exposure data were available. The surrogate level of exposure to each aromatic amine was calculated as the summed jobweight-month in each process in each job-year.

Results The mean ages of the non-BC and $\mathrm{BC}$ groups were 50 and 56 years and the durations of employment were 23 and 20 years, respectively. The smoking rate in both groups was $80 \%$. Significantly higher rates of gross hematuria $(70 \%)$ and cystitis $(70 \%)$ were identified in the BC group's past medical histories. In the $\mathrm{BC}$ group, the surrogate levels of exposure to OT were higher than those of exposures to other aromatic amines. The surrogate levels were high in the job processes of filtering, rinsing, drying and packing products.

Conclusions The subjects with BC were associated with a high surrogate level of exposure to OT. OT-exposed workers with past histories of gross hematuria and cystitis need a careful follow-up.

\section{P.2.21 OCCUPATIONAL PESTICIDE EXPOSURE ASSOCIATED WITH A REDUCTION OF RESPIRATORY PARAMETERS IN ETHIOPIAN FARM WORKERS}

${ }^{1}$ Beyene Negatu, ${ }^{2}$ Kromhout Hans. ${ }^{1}$ Pesticide Registration and Control Department, Addis Ababa, Ethiopia; ${ }^{2}$ Institute for Risk Assessment Sciences, Utrecht, The Netherlands

\subsection{6/OEM-2019-EPI.254}

There are reports of unsafe occupational handling and use of pesticide in recently intensifying commercial farming systems in Ethiopia. Very few studies reported the effect of occupational pesticide exposure on smaller airway respiratory parameters of farm workers from low and middle-income counties. A cross-sectional spirometry survey was carried out on randomly selected 387 subjects with an objective of investigating whether occupational exposure to pesticides is associated with a reduction in values of smaller airway respiratory parameters. 206 occupationally exposed (142 male pesticide applicators and 64 female re-entry workers) selected from commercial farms and 180 occupationally un-exposed male and female individuals involved in the survey. After controlling for potential confounders, pesticide exposure in male study subjects was associated with reduced respiratory parameters of Forced Expired Flow at 25\% of vital capacity $\left(\right.$ FEF25\%) $\quad(1 / \mathrm{s}) \quad\left[\beta=-0.69 \quad\left(95 \% \quad\right.\right.$ CI $\left.\left.-1.11_{-}-0.27\right)\right]$ and Forced Expired Flow at $75 \%$ of vital capacity (FEF75\%) (l/s) $\left[\beta=-0.49\right.$ (95\% CI $\left.-0.78 \_-0.20\right]$. Also a reduction in respiratory parameters of Forced Expired Flow at 50\% of vital capacity (FEF50\%) [ $\beta=-0.52 \quad\left(95 \%\right.$ CI $\left.\left.-0.95 \_-0.09\right)\right]$ was seen among exposed female subjects. The study indicated occupational exposure to pesticides is associated with a reduction of respiratory parameters of smaller airways in both male and female farm workers. Further longitudinal studies on respiratory parameters are warranted in Ethiopian farm workers.

\section{P.2.23 ADVERSE CHILDHOOD EXPERIENCES (ACE) IN EARLY LIFE AND THE RISK OF CHILDHOOD ASTHMA: A DANISH NATIONWIDE COHORT STUDY}

1,2Kathrine Pape* ${ }^{1}{ }^{1}$ Camilla S Sejbæk,${ }^{2,3}$ Niklas W Andersson, 1,4 Karin S Hougaard ${ }^{5,6}$ Cecilie Svanes, ${ }^{7}$ Xiaogin Liu, ${ }^{8}$ Whitney Cowell, ${ }^{8}$ Rosalind Wright, ${ }^{1,2}$ Vivi Schlünssen. ${ }^{1}$ National Research Center for The Working Environment, Copenhagen O, Denmark; ${ }^{2}$ Section for Environmental and Occupational Medicine, Department of Public Health, Aarhus University, Aarhus, Denmark; ${ }^{3}$ Department of Epidemiology Research, Statens Serum Institut, Copenhagen, Denmark; ${ }^{4}$ Section of Environmental Health, Institute of Public Health, University of Copenhagen, Copenhagen, Denmark; ${ }^{5}$ Center for International Health, Department of Global Public Health and Primary Care, University of Bergen, Bergen, Norway; ${ }^{6}$ Department of Occupational Medicine, Haukeland University Hospital, Bergen, Norway; ${ }^{7}$ NCRR-The National Center for Register-based Research, Aarhus University, Aarhus, Denmark; ${ }^{8}$ Department of Pediatrics and Environmental Medicine and Public Health, Icahn School of Medicine at Mount Sinai, New York City, USA

\subsection{6/OEM-2019-EPI.255}

Objective A link between adverse childhood experience (ACE) in early life and subsequent asthma is suggested, but existing studies are often based on parent-reported data for both exposure and outcome. We aimed to examine the association of ACE in early life (bereavement, parental chronic somatic illness, or psychiatric illness/suicide attempt) with childhood asthma, using registry information of exposures and outcome.

Methods We used registry data of 466556 children born in Denmark, 1997-2004. ACE and asthma diagnosis or medication was obtained from the Danish National Patient or Prescription Registry. We used multinomial logistic regression to examine the association between ACE in early life and phenotypes of childhood asthma, which we empirically estimated using group-based trajectory modeling. We adjusted for year of birth, maternal age, smoking, place of living, parity, parental education and atopic status; we imputed missing data using multiple imputations with chained equations.

Results We identified four asthma trajectories: early onset (before age 3) transient asthma, late onset (3 years or later) asthma, early onset persistent asthma, and never/infrequent asthma. Girls exposed to at least one ACE before the age of 2 years, compared to the non-exposed, had higher odds of being assigned to the early-onset transient asthma group (odds ratio (OR) 1.13 [95\% Confidence Interval (CI): 1.04-1.24]), 